\title{
Levantamento sobre pontos de despejo de esgoto e percepção de banhistas sobre balneabilidade do rio Turiaçu no município Santa Helena-MA
}

Survey of sewage disposal points and bathers' perception of the Turiaçu River in the municipality of Santa Helena-MA

Encuesta sobre los puntos de evacuación de aguas residuales y la percepción de los bañistas del río Turiaçu en el municipio de Santa Helena-MA

Carlos Wilker Souza Pacheco ORCID: https://orcid.org/0000-0001-8260-1464 Universidade Federal do Maranhão, Brasil E-mail: wilkercarlos43@gmail.com Hilton Costa Louzeiro ORCID: https://orcid.org/0000-0003-1567-8183 Universidade Federal do Maranhão, Brasil E-mail: louzeiro.hc@gmail.com

Mikele Candida Sousa de Sant'Anna ORCID: https://orcid.org/0000-0002-9023-0154 Universidade Federal do Maranhão, Brasil E-mail: mikelecandida@gmail.com

Danilo Francisco Corrêa Lopes ORCID: https://orcid.org/0000-0001-8711-3881 Universidade Federal do Maranhão, Brasil E-mail: lopesdanilocorrea7@gmail.com

Nubia Fernanda Marinho Rodrigues

ORCID: https://orcid.org/0000-0002-5156-8128

Universidade Federal do Maranhão, Brasil E-mail: rnubiads@gmail.com

Paulo Roberto Barros Gomes

ORCID: https://orcid.org/0000-0002-4221-6577 Instituto Federal de Educação, Ciência e Tecnologia do Pará, Brasil E-mail: prbgomes@yahoo.com.br

Rômicy Dermondes Souza

ORCID: https://orcid.org/0000-0002-4477-453X Universidade Estadual do Sudoeste da Bahia, Brasil E-mail: dermondesromicy@gmail.com

Leandro Lima Carvalho

ORCID: https://orcid.org/0000-0003-3386-2769 Instituto de Ensino Médio e Superior Fancois Marie Arouet Ltda, Brasil

E-mail: leandrolimacarvalho75@gmail.com

Victor Elias Mouchrek Filho

ORCID: https://orcid.org/0000-0003-2855-7292

Universidade Federal do Maranhão, Brasil

E-mail: victor.mouchrek@ufma.br

Charbel Nagib Mouchrek

ORCID: https://orcid.org/0000-0002-9392-3474

Universidade Federal do Maranhão, Brasil E-mail: charbelnm@gmail.com

Núbia Regia de Almeida

ORCID: https://orcid.org/0000-0002-5235-7146 Instituto Federal de Educação, Ciência e Tecnologia do Pará, Brasil E-mail: rnubiads@yahoo.com

Jaqueline Pereira de Araújo

ORCID: https://orcid.org/0000-0003-0538-1490 Instituto Federal de Educação, Ciência e Tecnologia do Pará, Brasil E-mail: araujojaqueline12@outlook.com

\section{Resumo}

A balneabilidade é um parâmetro importante para garantir a qualidade do rio para atividades recreativas, sendo uma ferramenta que possibilita minimizar riscos à saúde humana. A bacia hidrográfica do rio Turiaçu é importante, uma vez 
que atravessa o município de Santa Helena e, até onde se sabe, não há dados relacionados à balneabilidade neste município. Portanto, este trabalho teve como objetivo identificar os pontos de banhos e despejo de esgoto às margens do rio Turiaçu através de pesquisa in loco. Além disso, este trabalho aborda uma pesquisa quali-quantitativa realizada com a população do município de Santa Helena para identificar a balneabilidade do rio Turiaçu. Foram identificados 8 locais utilizados para banho e 3 pontos de despejo de esgoto. Alguns pontos de banho estão próximos a pontos de despejo de esgoto, e, visto que as pessoas utilizam os locais de banho com muita frequência, programas de monitoramento da balneabilidade se fazem necessário.

Palavras-chave: Balneabilidade; Rio Turiaçu; Locais de banho.

\begin{abstract}
Balneability is an important parameter to ensure the quality of the river for recreational activities, being a tool that makes it possible to minimize risks to human health. The watershed of the Turiaçu River is important, since it crosses the municipality of Santa Helena and, as far as is known, there is no data related to Balneability in this municipality. Therefore, this work aimed to identify the bathing points and sewage dump on the banks of the Turiaçu river through in loco research. In addition, this work addresses a qualitative-quantitative research carried out with the population of the municipality of Santa Helena to identify the Balneability of the Turiaçu river. Eight bathing sites and three sewage disposal points were identified. Some bathing spots are close to sewage dump points, and, since people use bathing sites very often, monitoring programs for Biodegradability are necessary.
\end{abstract}

Keywords: Balneability; Turiaçu River; Bathing places.

\title{
Resumen
}

La Balneabilidad es un parámetro importante para garantizar la calidad del río para actividades recreativas, siendo una herramienta que posibilita minimizar riesgos a la salud humana. La cuenca hidrográfica del río Turiaçu es importante, ya que atraviesa el municipio de Santa Helena y, hasta donde se sabe, no hay datos relacionados con la estabilidad del agua en este municipio. Por lo tanto, este trabajo tuvo como objetivo identificar los puntos de baños y desagüe a orillas del río Turiaçu a través de investigación in situ. Además, este trabajo aborda una investigación quali-cuantitativa realizada con la población del municipio de Santa Helena para identificar la estabilidad del río Turiaçu. Se identificaron 8 lugares utilizados para baño y 3 puntos de desagüe. Algunos puntos de baño están cerca de los puntos de vertido de aguas residuales, y, puesto que las personas utilizan los sitios de baño con demasiada frecuencia, programas de monitoreo de la Balneabilidad se hacen necesarios.

Palabras clave: Balneabilidad; Río Turiaçu; Sitios de baño.

\section{Introdução}

O Brasil é um país rico em recursos hidrográficos. Estima-se que o país abriga $12 \%$ da água doce do planeta, tendo a maior concentração de produção hídrica presente na região amazônica (Barbieri \& Gomes, 2004). No entanto, o aumento da população somado às ocupações urbanas desordenadas e à falta de saneamento básico podem causar graves problemas sanitários a estes recursos hídricos, afetando as espécies presentes nos rios e lagos e, também, as pessoas que se beneficiam destes locais (Adelodun et al., 2021; Shakoor et al., 2017; Ternus et al., 2011). A qualidade sanitária dos recursos hídricos é um assunto de total importância nos tempos atuais, visto que a crescente degradação de rios e lagos pelas ações humanas podem reduzir a disponibilidade de água potável, além de destruir inúmeros ecossistemas (Barry et al., 2019; Duan et al., 2017; Warner, 1991).

A balneabilidade é um dos parâmetros utilizado para garantir a qualidade da água, pois ela define se a água está apropriada para atividades recreativas. Portanto, a balneabilidade significa segurança aos banhistas, haja vista a possibilidade de os usuários, em contato com a água, terem chances de ingerir quantidades consideráveis deste líquido (CONAMA, 2001). Esta ingestão representa um risco alto de contaminação microbiana, visto que águas de rios, lagoas e afins possuem a presença de microrganismos patogênicos e não patogênicos (Medeiros et al., 2014; Phiri et al., 2021). Segundo a resolução do CONAMA nº 274, a água, com relação a sua balneabilidade, pode ser categorizada como própria ou imprópria, sendo a água definida como imprópria àquela que apresentar quantidades de microrganismos, resíduos químicos e outras substâncias acima do permitido (CONAMA, 2001).

A bacia hidrográfica do rio Turiaçu apresenta área total de 14.014,08 km² e está distribuída em 19 munícios, onde residem no total, aproximadamente 491.571 habitantes (IBGE, 2021). Visto a quantidade de municípios beneficiados pelo rio Turiaçu, a análise de sua qualidade é algo indispensável, haja vista, a falta de dados publicados, relacionados à sua balneabilidade. 
Portanto, neste trabalho identificamos os principais pontos de banho e despejo de esgoto no rio Turiaçu, e mapeamos o conhecimento da população em torno da sua balneabilidade.

\section{Metodologia}

Trata-se de um estudo de caso descritivo de natureza qualitativa. Para tanto, delimitamos como área de estudo as margens do rio Turiaçu, mais especificamente o percurso que corta o município de Santa Helena, no Maranhão. Estas especificações gráficas estão presentes na figura 1. Na primeira parte da pesquisa identificamos os pontos de banhos e os pontos de despejo de esgoto por meio de visita in loco. Para identificar cada ponto, utilizamos o aplicativo de GPS Google Maps para celular com sistema Android 8.

Na segunda parte, realizamos um estudo quali-quantitativo com a população que se encontrava ao longo das margens do Rio. Neste estudo, buscamos definir com os participantes o perfil demográfico (gênero, idade e escolaridade), entendimento sobre balneabilidade, frequência e tempo de contato com água do rio, percepção e preocupação com a qualidade da água, possíveis doenças de pele causadas pelo contato com as águas e lesões sofridas no rio, e por fim, coletamos sugestões dos participantes para melhorar as práticas de lazer e a segurança da água do rio. Pereira et al. (Pereira et al., 2018) considera importante realizar este tipo de estudo, pois ele possibilita gerar uma "riqueza de dados e informações de modo a contribuir com o saber na área de conhecimentos na qual for utilizada".

Figura 1 - Mapa do Maranhão com destaque ao município de Santa Helena ( $₫)$ e à Bacia Hidrográfica de Turiaçu (匹).

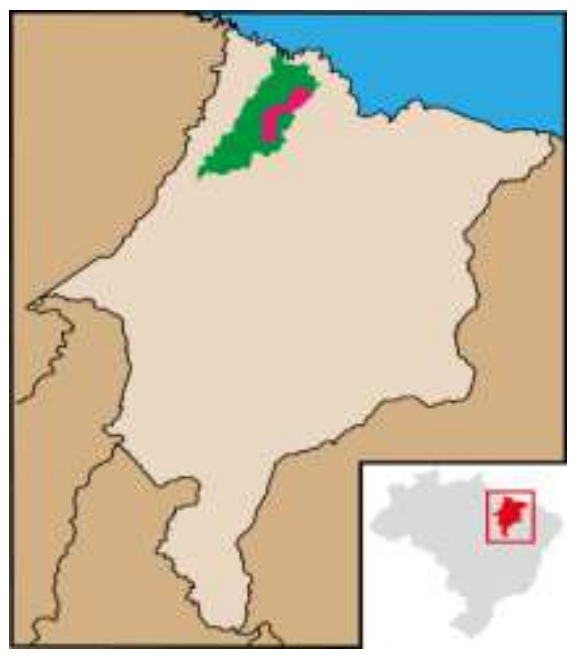

Fonte: Adaptado de IBGE (2021) e CODEVASF (2021).

\section{Resultados}

\subsection{Levantamento dos pontos de banho}

Na Figura 2 é apresentada a imagem de satélite do município de Santa Helena. Os 8 pontos de A à H, em vermelho (Figura 2), representam os locais de banho identificados na visita in loco. Durante a semana notamos, nestes locais, uma movimentação considerável de pessoas e, nos finais de semana, percebemos um fluxo maior. Estes locais de banhos são distribuídos nos seguintes bairros: Três no bairro Porta D’areia, 2 no bairro São Braz, 2 no bairro Centro e 1 próximo ao bairro Morada. Nestes pontos de banho, a partir da visita in loco, observamos a ausência de fiscalização ou organização da gestão municipal, além da falta de apoio técnico ou aparato de segurança. Identificamos um fluxo maior de pessoas nos pontos próximos a bares e similares. 
As marcações de 1 a 3, em amarelo (Figura 2), identificadas na visita in loco, representam as galerias municipais para escoamento de água de chuva, esgotos domésticos e outros fluídos oriundos da urbanização. Não notamos nenhum tipo de tratamento da água escoada, uma vez que foram construídas visando evitar alagamentos no perímetro urbano. No que diz respeito aos parâmetros de balneabilidade, não encontramos dados de coleta para o município em estudo.

Figura 2 - Imagem de satélite do município de Santa Helena: (A), (B), (C), (D), (E), (F), (G) e (H) Localização dos principais pontos utilizados por banhistas; (1), (2) e (3) Localização de galerias feitas pelo município para escoar esgoto.

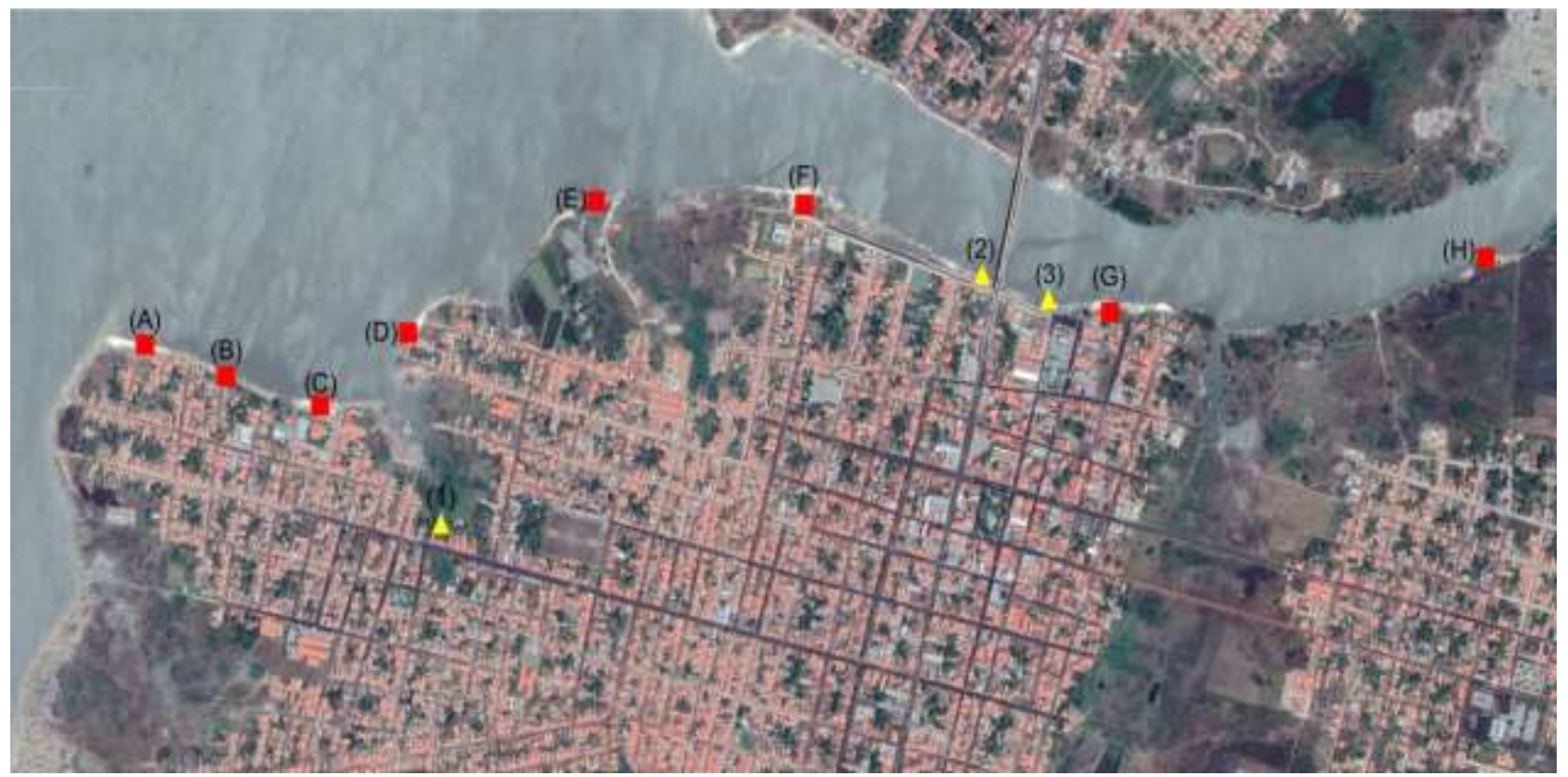

Fonte: Adaptação Google Maps (2019).

\subsection{Dados quali-quantitativos}

\subsubsection{Perfil dos entrevistados}

Com o objetivo de traçar o perfil demográfico dos entrevistados, eles foram convidados a informar sua escolaridade, idade e gênero. No total, entrevistamos 30 pessoas. Identificamos que a maioria dos entrevistados continha o ensino médio completo e idade entre 19 e 26 anos. Não observamos diferenças significavas em relação ao gênero. Os dados detalhados podem ser visualizados na Figura 3. 
Figura 3 - Dados demográficos dos entrevistados.

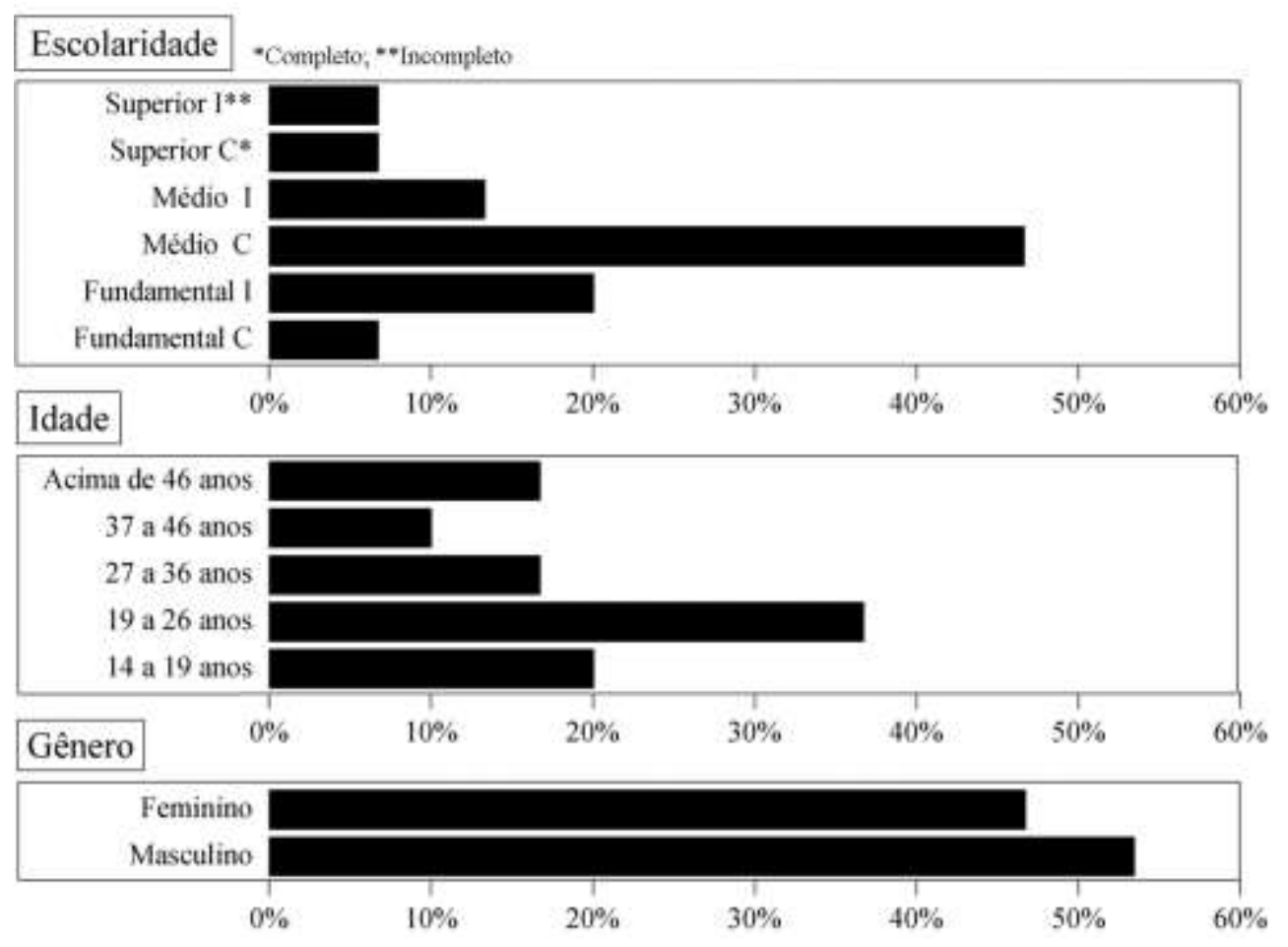

Fonte: Autores (2021).

\subsubsection{Entrevistas sobre balneabilidade}

Na segunda parte do questionário, buscamos traçar o conhecimento dos entrevistados a respeito da balneabilidade. Na primeira pergunta, pedimos para que os entrevistados, de forma aberta, comentassem o seu entendimento no que concerne a palavra balneabilidade. A respostas estão presentes na Tabela 1. Dos entrevistados, 4 afirmaram desconhecer o significado da palavra, enquanto a maioria respondeu de forma simplista, comentando somente sobre a qualidade da água destinada a banhos e ignorando outras práticas em que se tenha contato direto e contínuo com a água.

Tabela 1 - Resposta dos entrevistados para a pergunta: “O que você entende por balneabilidade?".

\begin{tabular}{l|l}
\hline É sobre a qualidade da água para prática de esportes. & Se a água ta boa para banho. \\
\hline Não sei responder. & É a capacidade que um local tem de possibilitar banho. \\
\hline Qualidade da água destinada ao banho. & Áreas de lazer para banhistas às margens do rio Turiaçu. \\
\hline Qualidade da água para prática de lazer. & $\begin{array}{l}\text { É a capacidade que o local tem para banho ou outras atividades } \\
\text { em suas águas. }\end{array}$ \\
\hline É balneário. & Não sei responder. \\
\hline Qualidade das águas dos rios para tomar banho. & Tem a ver com a água para banho. \\
\hline Não sei responder com exatidão no momento. & $\begin{array}{l}\text { É a qualidade da água destina a recreação. Ou seja, própria para o } \\
\text { banho. }\end{array}$ \\
\hline É a qualidade da água. & Qualidade da água para o banho. \\
\hline Estado da água do rio, se é boa ou não. & Nunca ouvi a palavra. \\
\hline Se a água ta boa ou não para o banho. & Sobre se a água é boa ou não para banhar. \\
\hline Área própria para banhista. & Qualidade da água própria para o banho. \\
\hline
\end{tabular}

Fonte: Autores (2021).

Com relação ao contato com as águas do rio Turiaçu, $84.3 \%$ afirmaram ter contato frequente, enquanto $16.7 \%$ relataram não ter o hábito de ir ao rio. Aos entrevistados que informaram contato frequente com o rio, realizamos alguns questionamentos que estão presentes em detalhes na Figura 4. Com relação ao tempo de contato com o rio, observamos que a maioria das pessoas 
utiliza por longos períodos, entre 30 a 240 minutos. Quando questionados sobre as atividades realizadas neste intervalo, as principais respostas foram banho e pesca. A lavagem de veículos nas margens do rio, também foi uma resposta frequente.

No que diz respeito à preocupação com a qualidade da água, $86.7 \%$ dos entrevistados, se mostraram apreensivos e preocupados com o descarte de resíduos nas margens do rio, enquanto $13.3 \%$ citaram não possuir esta preocupação. A maioria dos entrevistados classificou a qualidade da água como boa (36.7\%) e regular (36.7\%).

No questionário, também abordamos a temática de doença de pele, possivelmente associada ao contato com o rio. 70\% dos entrevistados citaram ter tido ou conhecer alguém que tenha tido micoses ou outro problema de pele em decorrência do contato com a água, enquanto 30\% relataram desconhecer sobre algum caso. Em relação às lesões sofridas no rio (figura 4), 80\% afirmaram terem se lesionado, enquanto $20 \%$ não sofreram nenhum acidente.

\section{Discussão}

Neste trabalho, determinamos os principais pontos de banhos e despejo de esgoto às margens do rio Turiaçu, além de coletar as opiniões da população a respeito da balneabilidade do rio Turiaçu. Em nossos resultados, identificamos 8 pontos de banho e 3 pontos de despejo de esgoto, estes últimos, próximos a locais de banho. Com o uso frequente da população aos locais de banho, por um longo período de tempo, faz-se necessário o acompanhamento e fiscalização das autoridades responsáveis nestes pontos de banhos a fim de garantir a segurança da população.

As pessoas que frequentam o rio regularmente costumam permanecer muito tempo, o que caracteriza risco sanitário, visto que alguns pontos de banho estão próximos aos locais de despejo de esgotos. A presença destes esgotos próximos aos locais de banho representa perigo sanitário, pois há risco de resíduos de medicamentos (Kosonen \& Kronberg, 2009), metais pesados (Chakarvorty et al., 2015) e microrganismos nocivos à saúde humana (Middleton \& Salierno, 2013). De acordo com os participantes, doenças de peles são aparentemente comuns nas margens do rio, o que possivelmente estão associadas ao despejo de resíduos em sua margem. Estas doenças de pele podem ter sido causadas por resíduos químicos ou pelas vastas gamas de microrganismos presentes na água contaminada (Lewis \& Gattie, 2002). Portanto, é necessário análise da qualidade de água em torno dos locais de banhos a fim de definir os pontos a segurança de sua utilização.

Apesar da aparente preocupação e conhecimento das questões sanitárias do rio, algumas pessoas realizam lavagens de veículos às suas margens, e que, somado ao despejo de esgoto doméstico, potencializa o problema sanitário. Os resíduos oriundos da lavagem de veículos geralmente incluem fósforo, nitrogênio, surfactantes, matéria orgânica, metais pesados, resíduos de hidrocarbonetos derivados do petróleo, entre outros, que quando descartados na água do rio agravam o problema sanitário (Rai et al., 2020).

Notamos também que os entrevistados conhecem pouco sobre balneabilidade. A falta de conhecimento científico confiável, tanto por parte da população quanto das autoridades, é considerada uma das principais barreiras para implementação de programas de gestão sanitária nas costas de rios e mares (Wagener, 2005). Neste sentido, sugerimos campanhas, de divulgação de conhecimento, apropriadas a respeito da conservação das águas do rio Turiaçu.

Por fim, com base nas respostas qualitativas, a maioria dos participantes da pesquisa concorda que para tornar as práticas de lazer melhores e mais seguras é necessária a limpeza da margem do rio. Todavia, somente limpeza periódica não é o suficiente para garantir segurança sanitária a longo prazo. Sugerimos também, além da limpeza das margens, a correta sinalização dos locais de banhos indicando os locais próprios para lazer e, principalmente, o desenvolvimento de um programa de monitoramento da balneabilidade por um órgão público competente (Morais \& Silva, 2010). Este monitoramento é geralmente feito com base na quantificação de coliformes fecais, mais especificamente E. coli, todavia, análises complementares podem ser realizadas, sendo elas: pH, turbidez, DBO e OD (Medeiros et al., 2014). 


\section{Conclusão}

No município de Santa Helena há vários locais de lazer utilizados pelos moradores no rio Turiaçu, alguns pontos ficam muito próximos ao despejo de esgotos. O acompanhamento das áreas de banho deve ser realizado para reduzir potenciais problemas sanitários, haja vista a utilização frequente do rio pela população. Sugerimos que sejam criados programas de monitoramento da balneabilidade para garantir a segurança sanitária dos banhistas, além de propagação de conhecimento técnicocientífico sobre balneabilidade para a população. Para trabalhos futuros recomendamos avaliação química e microbiológica da água nos pontos de banho, apresentados neste estudo, e identificação dos pontos mais seguros para lazer.

\section{Referências}

Adelodun, B., Ajibade, F. O., Ighalo, J. O., Odey, G., Ibrahim, R. G., Kareem, K. Y., Bakare, H. O., Tiamiyu, A. G. O., Ajibade, T. F., Abdulkadir, T. S., Adeniran, K. A., \& Choi, K. S. (2021). Assessment of socioeconomic inequality based on virus-contaminated water usage in developing countries: A review. Environmental Research, 192(July 2020), 110309. https://doi.org/10.1016/j.envres.2020.110309

Barbieri, J. C., \& Gomes, J. de L. (2004). Gerenciamento de recursos hídricos no Brasil e no estado de São Paulo : um novo modelo de política pública. Cadernos EBAPE.BR, 2(3), 1-21.

Barry, D., Barbiero, C., Briens, C., \& Berruti, F. (2019). Pyrolysis as an economical and ecological treatment option for municipal sewage sludge. Biomass and Bioenergy, 122, 472-480. https://doi.org/10.1016/j.biombioe.2019.01.041

Chakarvorty, M., Dwivedi, A. K., Shukla, A. D., Kumar, S., Niyogi, A., Usmani, M., \& Pati, J. K. (2015). Geochemistry and magnetic measurements of suspended sediment in urban sewage water vis-à-vis quantification of heavy metal pollution in Ganga and Yamuna Rivers, India. Environmental Monitoring and Assessment, 187(9), 1-17. https://doi.org/10.1007/s10661-015-4794-x

CODEVASF. (2021). Turiaçu. codevasf.gov.br/area-de-atuacao/bacia-hidrografica/Turiaçu

Qualidade Da Água, Resolução CONAMA nº 274, de 29 de novembro de 2000256 (2001).

Duan, B., Zhang, W., Zheng, H., Wu, C., Zhang, Q., \& Bu, Y. (2017). Disposal situation of sewage sludge from municipal wastewater treatment plants (WWTPs) and assessment of the ecological risk of heavy metals for its land use in Shanxi, China. International Journal of Environmental Research and Public Health, 14(7). https://doi.org/10.3390/ijerph14070823

IBGE. (2021). Centro Subregional B ( $3 B$ ), São Luís Pinheiro, Norte Maranhense, Baixada Maranhense./cidades.ibge.gov.br/brasil/ma/santa-helena/panorama Kosonen, J., \& Kronberg, L. (2009). The occurrence of antihistamines in sewage waters and in recipient rivers. Environmental Science and Pollution Research, 16(5), 555-564. https://doi.org/10.1007/s11356-009-0144-2

Lewis, D. L., \& Gattie, D. K. (2002). Pathogen risks from applying sewage sludge to land. Environmental Science and Technology, 36(13), 287-293. https://doi.org/10.1021/es0223426

Medeiros, S. R. M. de, Carvalho, R. G. de, Souza, L. di, \& Barbosa, A. H. da S. (2014). Índice de qualidade das águas e balneabilidade no Riacho da Bica, Portalegre, RN, Brasil. Rev. Ambient. Água, 11(3), 711-729. https://doi.org/10.4136/1980-993X

Middleton, J. H., \& Salierno, J. D. (2013). Antibiotic resistance in triclosan tolerant fecal coliforms isolated from surface waters near wastewater treatment plant outflows (Morris County, NJ, USA). Ecotoxicology and Environmental Safety, 88, 79-88. https://doi.org/10.1016/j.ecoenv.2012.10.025

Morais, R. C. de S., \& Silva, C. E. da. (2010). Diagnóstico ambiental do balneário Curva São Paulo no rio Poti em Teresina, Piauí. Eng Sanit Ambient, 17(1), 41-50.

Pereira, A., Shitsuka, D., Parreira, F., \& Shitsuka, R. (2018). Metodologia da pesquisa científica. In Metodologia da Pesquisa Científica. https://repositorio.ufsm.br/bitstream/handle/1/15824/Lic_Computacao_Metodologia-Pesquisa-Cientifica.pdf?sequence=1. Acesso em: 28 março 2020.

Phiri, B. J., French, N. P., Biggs, P. J., Stevenson, M. A., Reynolds, A. D., Garcia-R, J. C., \& Hayman, D. T. S. (2021). Microbial contamination in drinking water at public outdoor recreation facilities in New Zealand. Journal of Applied Microbiology, 130(1), 302-312. https://doi.org/10.1111/jam.14772

Rai, R., Sharma, S., Gurung, D. B., Sitaula, B. K., \& Shah, R. D. T. (2020). Assessing the impacts of vehicle wash wastewater on surface water quality through physico-chemical and benthic macroinvertebrates analyses. Water Science, 34(1), 39-49. https://doi.org/10.1080/11104929.2020.1731136

Shakoor, M. B., Nawaz, R., Hussain, F., Raza, M., Ali, S., Rizwan, M., Oh, S. E., \& Ahmad, S. (2017). Human health implications, risk assessment and remediation of As-contaminated water: A critical review. Science of the Total Environment, 601-602, 756-769. https://doi.org/10.1016/j.scitotenv.2017.05.223

Ternus, R. Z., de Souza-Franco, G. M., Anselmini, M. E. K., Mocellin, D. J. C., \& dal Magro, J. (2011). Influence of urbanisation on water quality in the basin of the upper Uruguay River in western Santa Catarina, Brazil. Acta Limnologica Brasiliensia, 23(2), 189-199. https://doi.org/10.1590/s2179$975 \times 2011000200009$

Wagener, A. de L. . (2005). Constraints to the implementation of effective environmental management in coastal areas of developing countries. Anais Da Academia Brasileira de Ciencias, 77(4), 613-623. https://doi.org/10.1590/s0001-37652005000400004

Warner, R. F. (1991). Impacts of environmental degradation on rivers, with some examples from the hawkesbury-nepean system. Australian Geographer, 22(1), 1-13. https://doi.org/10.1080/00049189108703016 